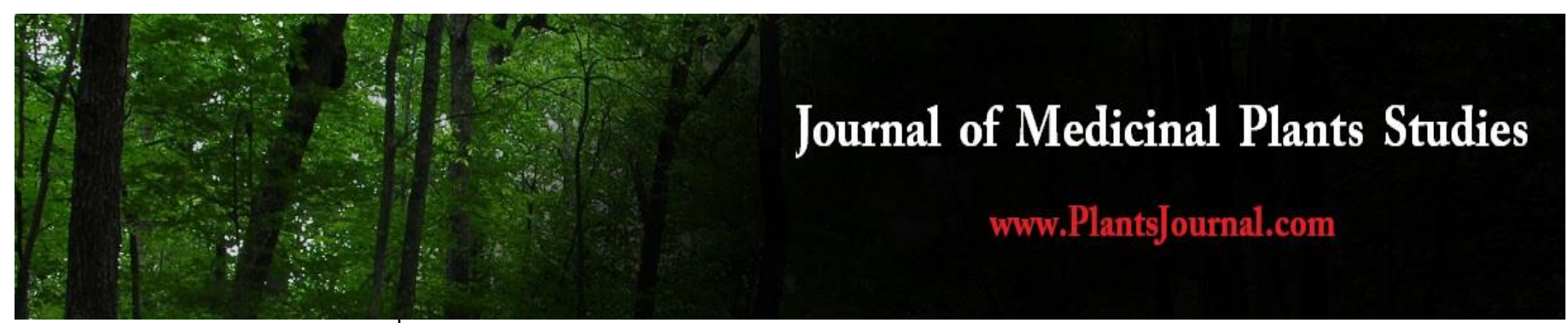

ISSN (E): 2320-3862 ISSN (P): 2394-0530

NAAS Rating: 3.53

JMPS 2018; 6(6): 64-67

(C) 2018 JMPS

Received: 12-09-2018

Accepted: 14-10-2018

Renu Joshi

Director,

studyofplants.com

Connecticut, USA
Correspondence

Renu Joshi

Director,

studyofplants.com

Connecticut, USA

\section{A Review on Colletotrichum spp. Virulence mechanism against host plant defensive factors}

\section{Renu Joshi}

\section{DOI: https://doi.org/10.22271/plants.2018.v6.i6b.02}

\section{Abstract}

Colletotrichum species are a group of pathogenic fungi that devastate farmers around the world and causing huge loses in crop production worldwide. The pathogen is the major causative agent of anthracnose disease that is affecting a wide range of crops. The disease is common in the tropics and subtropic regions but some species have been found to inhabit the Mediterranean climate. Control of Colletotrichum has proved to be a challenge due to its ability to develop new infection strategies that enables it to overcome the host inhibitory mechanisms. This paper therefore aimed at reviewing the virulence mechanisms of the pathogen to enable it to overcome the plant defensive mechanisms. From the different reviews it came out clearly that the pathogen infection encompasses the differentiation of specialized cell types that are associated with penetration, growth inside living host cells and colonizing with killing (bio trophy) and tissue colonization and destruction (necrotrophy). The fungi also produce some of the proteins and enzymes that aid in their penetration and attack of the host. Some of the enzymes that release by these species of fungi are cellulases that catalyses the degradation of host cell, pectin lytic enzyme, endo-polygalacturonases, protein kinases, glucanase and chitins. The fungi also secrete protein effectors that enable them reprogram the host cell immunity factors. Further virulence mechanism of Colletotrichum species is the power to modify the secondary antimicrobial compound the phenolics to less inhibitory compounds for example conversion of phaseollin to 1a-hydroxyphaseollone. Other contributing factors to the success of Colletotrichum attack on the host plants is the production of chemicals such as; colletotric acid, ergosterol, Colletotrichum and colletruncoic acid methyl ester.

Keywords: Colletotrichum, virulence, host plant defensive factors

\section{Introduction}

Colletotrichum spp. are the main contributory pathogen for anthracnose disease, a fungal disease devastating farmers and causing huge economic loses around the world. According to Dean et al. (414-430), Colletotrichum is ranked the eighth most devastating plant pathogenic fungi in the world. Genus Colletotrichum comprises of many species causing plant disease on a wide range of plants covering both the woody and herbaceous plants (Cannon 181-213). Occurrence and effects of anthracnose disease caused by Colletotrichum species is very common in the tropical and subtropical areas where the climatic conditions are warm and humid, but recent research has shown some high profile species of Colletotrichum surviving in the temperate regions and thus affecting the temperate crops. The fungi affect a wide range of plants ranging from fruits like citrus, mangoes, bananas, avocado, straw berries and coffee berries. The fungi also affect cereals including, maize, sorghum, sugarcane, and leguminous plants such as common bean (Phaseolus vulgaris), cow pea, pigeon pea among others (Dean et al. 414-430). The pathogen infects the plants and affecting the quality of the crop products fruits for export in tropical, sub-tropical and Mediterranean regions are the most affected leading to economic loses of over $80 \%$ in worst scenarios.

Most species of Colletotrichum are seed borne as well as soil borne. Their ascosporic may be spread by air while the conidia may be dispersed by water splash especially during the rainy seasons. The pathogen Colletotrichum is majorly known for anthracnose disease but also contributes to other plant diseases such as straw berry crown rot, banana crown rot, red rot in coffee berries and sugar cane as well as blotch in cowpea. The ability to cause this complex of diseases enhances its economic effects (Lenne 4-18). Colletotrichum causes anthracnose disease which exhibit a number of symptoms which include; sunken necrotic lesions on the 
leaves, fruits, stems and even roots, there are also crown and stem rots as well as seed blight (Lenne 4-18).

Colletotrichum infect the plants by attaching on the surface with the help of aspersorium (germinating spore), the aspersoria develop into a hyphae and squeezes itself through the plant cuticle into the epidermal cells (Liao et al. 262-270). The fungus grows and reproduce while in the host and produces host induce virulence effectors which aid their survival. During infection process the pathogen enter to consecutive stages the bio trophic phase where the inner cells and tissues are infected but do not show the symptoms. The infection then enters the necrotic phases where the pathogen colonizes the cells and the tissues leading the death of the plant cells and thus the necrotic lesions which can be observed externally (Liao et al. 262-270).

\section{Host plants and their distribution}

There are approximately 600 species of Colletotrichum affecting a round 3200 of plants monocots and dicots inclusive. Most of these Colletotrichum species are host specific though there are some arguments that the issue of host specificity is imperfect due to various number of factors (Cannon 181-213; O'Connell et al. 1060). For instance, most of the grass family are affected by Colletotrichum graminicola, C. graminicola has been an important pathogen affecting maize, rice and wheat production in the world. In USA alone $C$. graminicola has been reported to cause annual loses of over one billion dollars. Colletotrichum higginsianum affecting the brassica family mainly the kales, cabbages and broccoli leading to huge loses. Alfalfa have been affected by Colletotrichum trifolii while the legumes have been affected by Colletotrichum lindemuthianum (O'Connell et al. 1060). The effects of this fungal pathogen have a very huge economic impact worldwide.

\section{Host Plants Defensive Mechanisms}

Plants are the baseline of all the food chain offering a source of nutrients and energy to almost all organisms ranging from micro-organisms bacteria, fungi and viruses to macroorganisms such as insect and the larger group of vertebrates (Freeman et al. 1-12). Most of microorganisms are parasitic in nature and they attack the plants to get nutrients and in the process cause diseases. To counter the effects of pathogens plants have developed a number of structural, chemical and protein defence mechanisms (Freeman et al. 1-12). Thickened cell wall and the cuticle is the most common structural defensive component against most plant pests. Many studies have shown that plants produce phenylalanine ammonia lyase (PAL) an enzyme that counters the attack by fungi. Plants also reacts to Colletotrichum and other fungal infections by producing defensive chemical compounds (Siupp and Bailey 101-112; Heath 77-90). Among such chemical compounds are mainly phenolic in nature which includes; phytoalexins phase Olin, phaseolidin, phaseolinisoflavan, kievitone, merdicarp and pisatin (Bailey and Vincent 1789-1791; Siupp and Bailey 101-112; Hargreaves and Bailey 89-100; Heath 77-90). These compounds reduce severity of $C$. lindemuthianum by mechanisms such as disrupting spores and the hyphal contents while inducing resistance of host plants to the fungal pathogen (Bailey and Vincent 1789-1791).

Plants also produce volatile organic compounds (VOCs) which confer resistance against the fungal pathogen $\mathrm{C}$. lindemuthianum (Quintana-Rodriguez et al. 250-260). Some VOCs produced by common bean include limonene, linalool, nonanal, methyl salicylate and methyl jasmonate (Quintana-
Rodriguez et al. 250-260). VOCs inhibit pathogen development by hindering conidial germination of the fungus (Neri et al. 30-35). Production of reactive oxygen species (ROS) and hydrogen peroxide are another most rapid form of plant defence against the pathogens. These compounds are used in the biosynthesis of lignin to enhance the strength of the epidermial cells and to synthesis of phenolics (Torres et al. 397-403). Plants also react hyper sensitively by increased production of mRNAs genes coding for Class 1 acid chitinase and protease inhibitors (Vargas et al. 1342-1358). For instance, a research to confirm this genes upregulation on maize showed that when the plants are infected by pathogens mRNAs levels of ZmPRI, ZmPRI4b, Zm PRI5, chitinase (ZmChit) and protease inhibitor (ZmWind). These compounds were produced around the infected areas and served to inhibit further attack by the pathogen (Vargas et al. 1342-1358). Some plants also produce polygalacturonase inhibitor proteins (PGIPs) to impede the penetration of the Colletotrichum species through the plant cell walls. PGIPs are considered as among the earliest defensive compounds released just on the onset of pathogen attacks (Howell and Davis 5-15).

\section{Colletotrichum infection mechanism and pathogenicity}

Colletotrichum species use different strategies to successfully invade the plants despite of their antimicrobial properties. These strategies comprise of intracellular hemibiotrophy and subcuticular necrotrophy (Vargas et al. 1342-1358) The pathogen use a multistage bitrophic infection technique through development of an appressoria that aid in attachment, chemical and enzyme release for cuticle degradation and penetration and later leading to necrotrophy (O'Connell et al. 1060). It has also been found that for successful infection and ability to cause anthracnose disease by Colletotrichum is aided by production of phytotoxic metabolites (García Pajón and Collado 426-431). Some of the phytotoxic metabolites that have been researched on vary with the host plant and the Colletotrichum species. For instance, Colletotrichum capsici has been reported to produce the toxic metabolites colletotrichin, colletodiol, colletoketol, colletol and colletallol, which apart from increasing infection ability they also reduce seed germination, root and shoot growth as well as causing seedling mortality in chilli Capsicum annuum (García Pajón and Collado 426-431; Anand et al. 437-451). Colletotrichum gloeosporioides has been reported to release colletotric acid in the stem of Artemisia mongolica (Zou et al. 1529-1530), and ergosterol in the stems of Artemisia annua (Lu et al. 63-67). Colletotrichum nicotianae releases colletotrichin in tobacco Nicotiana tabacum while Colletotrichum truncatum produces meso- and D (-)-butane2, 3-diol, 2-hydroxymethylhexa-2, 4-dienol and colletruncoic acid methyl ester in soybean G. max (García Pajón and Collado 426-431).

The fungal pathogens also exudate macerating enzymes that enable them to successfully infect host plants (O'Connell et al. 1610; Peeran at al. 295-300). Colletotrichum species produce the enzyme cellulase that catalyses the degradation of host cell walls (Anand et al. 2008; Peeran et al. 2014). Other enzymes produced by Colletotrichum species include pectinolytic enzyme, endo-polygalacturonases, protein kinases, glucanase and chitinase (Dickman 127-147; Anand et al. 437-451; Peeran et al. 295-300).

Common bean $P$. vulgaris reacts to Colletotrichum and other fungal infections by producing defensive chemical compounds (Siupp and Bailey 101-112). Among such 
chemical compounds are the phytoalexins phaseolin, phaseolidin, phaseolinisoflavan, kievitone, merdicarp and piston (Heath 77-90; Siupp and Bailey 101-112; Hargreaves and Bailey, 89-100). These compounds reduce severity of $C$. lindemuthianum by mechanisms such as disrupting spores and the hyphal contents while inducing resistance of host plants to the fungal pathogen (Bailey and Vincent 1789-1791).

Plants also produce volatile organic compounds (VOCs) which confer resistance against the fungal pathogen $C$. lindemuthianum (Quintana-Rodriguez et al. 250-260). Some VOCs produced by common bean include limonene, linalool, nonanaldehyde, methyl salicylate and methyl jasmonate (Quintana-Rodriguez et al. 250-260). VOCs inhibit pathogen development by hindering conidial germination of the fungus (Neri et al. 30-35). To counter these inhibitory factors Colletotrichum species have developed some mechanisms to modify plant antimicrobial metabolites for them to successfully infect the host plants (van den Heuvel and van Etten 327-339; Bailey and Vincent 1789-1791; van den Heuvel and Vollaard 103-108). For instance, $C$. lindemuthianum and Botrytis cinerea convert the phytoalexin phaseollin into 6a-hydroxyphaseollin and 7dihydroxyphaseollin; these newly formed chemical compounds show slight inhibitory effects to some races of Colletotrichum spp. (van den Heuvel and Glazener 125-137; van den Heuvel and Vollaard 103-108). Some Colletotrichum species, Fusarium solani ff.spp. phaseoli and the nonpathogenic fungus Cladosporium herbarum have developed the ability to convert phaseollin into 1ahydroxyphaseollone (van den Heuvel and Glazener 125-137). These compounds produced after interaction with the pathogenic microbes are less fungitoxic than the plant defense metabolite phaseollin (van den Heuvel and van Etten 327339; van den Heuvel and Vollaard 103-108).

Most plant pathogens including Colletotrichum species have been found to secrete protein effectors that reprogram host cell immunity thereby facilitating infection. Study by O'Connell et al. (1060) showed that Colletotrichum graminicola and $C$. higgisianum produce candidate secreted effectors (CSEPs) which are actually effector proteins.

\section{Control mechanisms}

Control of Colletotrichum species has been tricky due to the evolved mechanisms to counter the plant immunity factors. Chemicals have been used overtime to control the spread of the fungi but total control has been a challenge due to development of resistance by the pathogen (Munoz 11-15). Some of the commonly used fungicides that have been used to control Colletotrichum species include; propiconazole, bitertanole, hexaconazole, imazalii, carbendazin and thiabendazole. Some of the recent researches have shown that the use of plant extracts can help in controlling Colletotrichum spread. Alcohol and water extract from citrus lemon and piper betle have been found to be effective against the spread of Colletotrichum lindemuthianum (Amadioha 259-265). Development of tolerant varieties remains an option to curb the effects of Colletotrichum and improving on crop yields.

\section{Conclusion}

Colletotrichum species are devastating farmers around the world. The fungi have affected a range of crops ranging from farm orchards to leguminous crops and cereals. The pathogen cause anthracnose disease which affects the quality of the products. The effects of the disease have been increasing overtime due to development of penetration and infection mechanisms that counter the plant immune mechanisms. Some of the mechanisms that have been reported are the use of cell wall degrading enzymes, modification of antimicrobial phenolic compounds such as conversion of phaseollin into 1ahydroxyphaseollone a compound that that has less fun GU toxic activity giving room for the pathogen to penetrate and infect the plants leading to the development of the diseases.

Due to the modification mechanisms of the pathogen to attack the plants it's paramount to identify different and better way of managing the pests. There is need to come up with the tolerant varieties that can withstand the existence of the pathogen without affecting the yields and the quality of the products. Future strategies should also seek avenues of incorporating novel genes and chemical control to come up with the most effective Integrated Pest Management (IPM) technique that will help curb the spread of the pathogen.

\section{References}

1. Amadioha AC. "Evaluation of some plant leaf extracts against Colletotrichum lindemuthianum in cowpea." Acta Phytopathologica et Entomological Hungarian. 2003; 38.3-4:259-265.

2. Anand Theerthagiri, et al. "Production of cell wall degrading enzymes and toxins by Colletotrichum capsici and Alternaria alternata causing fruit rot of chillies." Journal of plant protection research. 2008; 48.4:437-451.

3. Bailey JA, Vincent GG. "Metabolism of phaseollin by Colletotrichum lindemuthianum." Phyto chemistry. 1974; 13.9:1789-1791.

4. Cannon PF, et al. "Colletotrichum-current status and future directions." Studies in mycology. 2012; 73:181213.

5. Dean Ralph, et al. "The Top 10 fungal pathogens in molecular plant pathology." Molecular plant pathology. 2012; 13.4:414-430.

6. Dickman MB. Colletotrichum. In Fungal Pathology. Springer Netherlands, 2000, 127-147.

7. Freeman Brian C, Gwyn A. Beattie. "An overview of plant defenses against pathogens and herbivores." The Plant Health Instructor, 2008, 1-12

8. García-Pajón CM, Isidro G Collado. "Secondary metabolites isolated from Colletotrichum species." Natural product reports. 2003; 20.4:426-431.

9. Hargreaves JA, JA Bailey. "Phytoalexin production by hypocotyls of Phaseolus vulgaris in response to constitutive metabolites released by damaged bean cells." Physiological Plant Pathology. 1978; 13.1:89-100.

10. Heath Michèle C. "Hypersensitive response-related death." Programmed cell death in higher plants. Springer, Dordrecht, 2000, 77-90.

11. Howell Jason T, MR Davis. "Plant defense mechanisms against fungal pathogens: polygalacturonase inhibitor proteins." Canadian journal of plant pathology. 2005; 27.1:5-15.

12. Lenné JM. "Some major plant diseases." Plant Pathologist's Pocketbook, 2002, 4-18.

13. Liao CY, et al. "Formation of highly branched hyphae by Colletotrichum acutatum within the fruit cuticles of Capsicum spp." Plant pathology. 2012; 61.2:262-270.

14. Lu Hong, et al. "New bioactive metabolites produced by Colletotrichum sp., an endophytic fungus in Artemisia annua." Plant science. 2000; 151.1:67-73. 
15. Munoz F Romero. "Effect of different fungicides in the control of Colletotrichum acutatum, causal agent of anthracnose crown rot in strawberry plants." Crop Protection. 2002; 21.1:11-15.

16. Neri Fiorella, et al. "Fungicidal activity of plant volatile compounds for controlling Monilinia laxa in stone fruit." Plant Disease. 2007; 91.1:30-35.

17. O'Connell Richard J, et al. "Lifestyle transitions in plant pathogenic Colletotrichum fungi deciphered by genome and transcriptome analyses." Nature genetics. 2012; 44.9:1060.

18. Peeran Mohammed Faisal, Prabakar Kuppusami, And Raguchander Thiruvengadam. "Pathogenesis of Colletotrichum lindemuthianum the incitant of anthracnose disease in beans mediated by macerating enzymes." The Bio scan. 2014; 9.1:295-300.

19. Quintana-Rodriguez Elizabeth, et al. "Plant volatiles cause direct, induced and associational resistance in common bean to the fungal pathogen Colletotrichum lindemuthianum." Journal of Ecology. 2015; 103.1:250260.

20. Siupp RA, Bailey JA. "The fungitoxicity of isoflavanoid phytoalexins measured using different types of bioassay." Physiological Plant Pathology. 1977; 11.1:101-112.

21. Torres, Miguel Angel, and Jeffery L. Dangl. "Functions of the respiratory burst oxidase in biotic interactions, abiotic stress and development." Current opinion in plant biology. 2005; 8.4:397-403.

22. Van den Heuvel J, HD. Van Etten. "Detoxification of phaseollin by Fusarium solani f. sp. phaseoli." Physiological Plant Pathology. 1973; 3.3:327-339.

23. Van den Heuvel J, PJ Vollaard. "Metabolism of phaseollin by different races of Colletotrichum lindemuthianum." Netherlands Journal of Plant Pathology. 1976; 82.3:103-108.

24. Van den Heuvel, Johannes, Judy A. Glazener. "Comparative abilities of fungi pathogenic and nonpathogenic to bean (Phaseolus vulgaris) to metabolize phaseollin." Netherlands Journal of Plant Pathology. 1975; 81.4:125-137.

25. Vargas Walter A, et al. "Plant defense mechanisms are activated during biotrophic and necrotrophic development of Colletotricum graminicola in maize." Plant physiology. 2012; 158.3:1342-1358.

26. Zou WX, et al. "Metabolites of Colletotrichum gloeosporioides, an endophytic fungus in Artemisia mongolica." Journal of Natural Products. 2000; 63.11:1529-1530. 